\title{
Diseño y análisis de \\ una tarea enmarcada \\ en la Estadística Cívica
}

\author{
Lorena Guglielmone
}

\section{Resumen}

Este trabajo forma parte de la primera fase de una investigación de diseño para la que se elaboró una tarea destinada a estudiantes universitarios. La tarea diseñada aborda un fenómeno social, multivariado y complejo que es analizado a través de gráficos de burbujas dinámicos. El diseño de la tarea y el análisis del contenido se centró en las facetas de la Estadística Cívica. Las ideas estadísticas fundamentales vinculadas a la misma corresponden a las nociones de datos, distribución, variación y correlación. El análisis de la tarea denota la potencia conceptual y de fundamentos que puede enriquecer a una enseñanza orientada a formar ciudadanos y futuros profesionales estadísticamente cultos.

Palabras clave: Educación Estadística, Estadística Cívica, Alfabetización estadística, Gráficos de burbujas dinámicos, Análisis de contenido. Keywords: Statistical Education, Civic Statistics, Statitical Literacy, Dynamic bubble graphics, Content analysis.

\begin{abstract}
This work is part of the first phase of a design investigation for which a task was developed for university students. The designed task addresses a complex, multivariate, social phenomenon that is analyzed through dynamic bubble charts. The design of the task and the content analysis focused on the facets of Civic Statistics. The fundamental statistical ideas linked to it correspond to the notions of data, distribution, variation and correlation. Task analysis denotes the conceptual and fundamental power that can enrich a teaching aimed at forming citizens and future statistically literacy professionals.
\end{abstract}

Nombre del trabajo: Diseño de una tarea enmarcada en la Estadística Cívica Nombre y apellido de la autora: Lorena Guglielmone

Dirección completa: Facultad de Ciencias de la Administración, Universidad Nacional de Entre Ríos, Monseñor Tavella 1424, Concordia, Entre Ríos.

Lugar de trabajo: Universidad Nacional de Entre Ríos.

Teléfono de contacto: 03454231400 


\section{Introducción}

El abordaje de los problemas de las sociedades modernas requiere la comprensión de fenómenos multivariados y complejos y esa comprensión debe estar vinculada a la evidencia empírica. Hoy en día, la posibilidad de trabajar con grandes bases de datos de libre acceso - provistas por organismos oficiales-, facilita a los ciudadanos la participación de manera informada y competente en los debates públicos sobre temas de relevancia social, condición necesaria para el funcionamiento de la democracia (Engel, 2019). Afortunadamente, existen herramientas digitales que permiten, por ejemplo, realizar visualizaciones dinámicas de conjuntos de datos sociales, facilitando el análisis de fenómenos complejos.

Tal como afirman Gal (2019) y Engel (2019), las fake news, la desinformación e ignorancia son amenazas para la vida en democracia. Es el conocimiento basado en evidencia el que permite que las personas tomen conciencia sobre las injusticias y las desigualdades sociales. En un mundo cada vez más complejo, la participación de los ciudadanos es un recurso esencial para intervenir en la formulación de políticas públicas y en la toma de decisiones a nivel local, nacional e internacional (Engel, 2019).

\section{Marco de referencia}

La revolución de los datos impone al sistema educativo el gran desafío de formar ciudadanos que razonen y piensen estadísticamente para comprender la información vinculada a problemas sociales y actuar en consecuencia (Tauber, 2021). La participación de los ciudadanos en la sociedad requiere de habilidades vinculadas a la evaluación de información estadística sobre fenómenos de relevancia social. Desde ese lugar, presentamos una tarea que busca promover la alfabetización y el pensamiento estadístico a través del análisis de datos dinámicos sobre fenómenos complejos y cercanos al contexto profesional de los estudiantes.
La Alfabetización Estadística y la Estadística Cívica son los constructos en los que se fundamenta la tarea presentada. Al primero de ellos Gal (2004), lo define desde dos componentes interrelacionados: a) la habilidad para interpretar y evaluar críticamente la información estadística, argumentar sobre un conjunto de datos o sobre fenómenos estadísticos que pueden encontrar en diversos contextos $y, b$ ) la habilidad para discutir o comunicar reacciones sobre la información estadística, tales como la comprensión de su significado, opiniones sobre las implicaciones de dicha información, o preocupaciones sobre la validez de las conclusiones dadas. Por su parte, Engel (2019) introduce la Estadística Cívica como una subdisciplina de la Alfabetización Estadística, centrada en el tratamiento de temas de relevancia social y «fundamental para el interés público en relación con el bienestar social y económico de todos los ciudadanos y el funcionamiento de la democracia» (p. 16). Además del conocimiento básico, las estadísticas referidas a fenómenos sociales requieren de conocimientos que se ubican en la intersección de la Estadística, las Ciencias Sociales y la Educación, por lo que presentan características diferentes a las de otras estadísticas (Engel, 2019). Estas incluyen:

- Fenómenos multivariados: Los datos provienen de estudios observacionales o de encuestas por lo que suele ser muy difícil identificar de manera confiable las relaciones de causa y efecto. La descripción y comprensión de los fenómenos sociales involucra distintas variables que están correlacionadas, que interactúan entre sí, o que presentan relaciones no lineales.

- Datos agregados: En general, las estadísticas sobre la sociedad suelen incluir datos agrupados de diferentes maneras, por ejemplo, por género (hombres vs. mujeres). Las conclusiones por nivel de agregación pueden variar cuando los datos se agregan o desagregan dentro de subgrupos.

- Datos dinámicos: Los datos de estadísticas oficiales se suelen informar como tendencias a lo largo del tiempo y se actualizan a partir nuevos datos disponibles. 
- Textos ricos: El texto es el principal medio de comunicación de las estadísticas cívicas y, en general, incluye diferentes elementos como gráficos, tablas y medidas. Para estar informados, los ciudadanos deben ser capaces de comprender dichos elementos. - Visualizaciones innovadoras: la tecnología permite que los datos sociales puedan visualizarse de maneras sofisticadas y novedosas. Si bien esas visualizaciones habilitan análisis estadísticos muy ricos y potentes, necesitan de habilidades de acceso y manipulación de las imágenes obtenidas con tecnología.

Las características mencionadas muestran que el campo de la Estadística Cívica requiere de habilidades y conocimientos que van más allá de la aplicación de técnicas y procedimientos. La política social representa el punto neural de su abordaje, lo cual implica la voluntad de trabajar con datos, la capacidad de razonar, buscar y comunicarse con evidencia numérica y de propiciar la evaluación crítica y la reflexión de temas de relevancia social (Tauber, 2021).

Nicholson, Gal y Ridgway (2018) y Engel (2019) describen las facetas de la alfabetización estadística vinculadas a temas sociales, es decir, las facetas de la Estadística Cívica. Esos autores identifican tres grandes dimensiones que las agrupan: el compromiso y la acción, el conocimiento, y los procesos habilitadores. En la Tabla 1 mostramos las facetas que incluye cada dimensión y a continuación hacemos una descripción de cada una.

\begin{tabular}{|l|l|}
\hline Dimensión & Faceta (Fi) \\
\hline Compromiso y acción & $\begin{array}{l}\text { F1. Preparación para el compromiso social } \\
\text { F2. Evaluación crítica y reflexión } \\
\text { F3. Disposiciones }\end{array}$ \\
\hline Conocimiento & $\begin{array}{l}\text { F4. Estadística y riesgo } \\
\text { F5. Modelos y representaciones } \\
\text { F6. Metodología y proceso de investigación } \\
\text { F7. Extensiones en el área de estadísticas oficiales } \\
\text { F8. Conocimiento social contextual }\end{array}$ \\
\hline Procesos habilitadores & $\begin{array}{l}\text { F9. TIC e investigación de la información } \\
\text { F10. Núcleo cuantitativo } \\
\text { F11. Comprensión de textos y comunicación }\end{array}$ \\
\hline
\end{tabular}

\section{Tabla 1.}

Dimensiones y facetas de la Estadística Cívica.

\begin{abstract}
- Faceta 1 (F1): Preparación para el compromiso social. Esta faceta se encuentra en el corazón de la Estadística Cívica. La comprensión de qué se puede y debe hacer para debatir y abordar los problemas de índole social prepara a los ciudadanos y estudiantes para el compromiso social.
\end{abstract}

- Faceta 2 (F2): Evaluación crítica y reflexión. La evaluación crítica y la reflexión deben transformarse en una rutina para la utilización de datos, por más que estos provengan de fuentes confiables.

- Faceta 3 (F3): Disposiciones. Las actitudes de los ciudadanos frente a fenómenos de carácter social, 
no puede dejar de considerarse. Dichas actitudes influyen en el compromiso social tanto de manera positiva como negativa. La ignorancia de la evidencia empírica, la aceptación acrítica de la información o la creencia de que solo los expertos pueden entender los fenómenos sociales, son actitudes problemáticas que interfieren en la comprensión. - Faceta 4 (F4): Estadística y riesgo. En esta faceta se encuentran muchas de las ideas que se enseñan en los cursos introductorios de Estadística en el nivel superior, como ser: muestreo, población y representatividad, variabilidad, distribuciones, asociación y correlación, regresión, señal y ruido, entre otras.

- Faceta 5 (F5): Modelos y representaciones. La modelización de fenómenos sociales muchas veces requiere de modelos cualitativamente diferentes dependiendo del área en la que se quiere analizar el fenómeno. Por ejemplo, es de esperar que un economista y un sociólogo usen modelos distintos para estudiar la «pobreza», y los ciudadanos deben ser capaces de identificar y comprender esos modelos, así como las suposiciones básicas que subyacen a los mismos.

En ese sentido, la comprensión y evaluación crítica de representaciones y visualizaciones sofisticadas representa una competencia fundamental para entender fenómenos multivariados y complejos.

- Faceta 6 (F6): Metodología y proceso de investigación. Los métodos de investigación y algunas habilidades de procedimiento presentan tanto fortalezas como debilidades que los ciudadanos deben conocer y comprender. Por ejemplo, las vinculadas a métodos cuantitativos como la realización de encuestas, estudios observacionales y cuasi experimentos, que incluyen temas como: sesgo, variabilidad, aleatorización, entre otros.

- Faceta 7 (F7): Extensiones en el área de estadísticas oficiales. Las estadísticas oficiales que provienen de organismos nacionales (INDEC, BA Data, Yverá, etc.) e internacionales (Naciones Unidas, Banco Mundial, EuroStat, etc.), son fuentes de evidencia basadas en datos sobre temas de relevancia social. Sin embargo, muchas de las ideas claves que se utilizan reciben poca atención en los cursos de estadística, como ser las referidas al diseño de encuestas y los problemas de falta de respuesta o sesgo de repuesta, la operacionalización de variables, los problemas de medición que afectan la confiabilidad y validez de los datos, la definición y significado de metadatos, etc.

- Faceta 8 (F8): Conocimientos social contextual. La estadística se basa en la modelización de fenómenos, pero para poder modelar es necesario tener una comprensión básica del contexto de dichos fenómenos. El conocimiento social contextual incluye conocimientos generales relacionados a diferentes áreas como: economía (producto bruto interno, deuda nacional, recursos, etc.), demografía, derechos humanos, historia, geografía, geopolítica, entre otras. Una ventaja es que se pueden buscar explicaciones y análisis alternativos basados en el conocimiento de posibles covariables.

- Faceta 9 (F9): Tecnologías de la información y la comunicación (TIC) e investigación de la información. Muchos proveedores de estadísticas oficiales ponen a disposición del público bases de datos de libre acceso, lo cual requiere de conocimientos y habilidades de manejo de TIC. Para manipular los datos es necesario descargarlos e importarlos al software adecuado. Probablemente deban limpiarse, ordenarse, transformarse, agregarse 0 desagregarse, y esas habilidades deben promoverse a través de propuestas de enseñanza.

- Faceta 10 (F10): Núcleo cuantitativo. Las habilidades cuantitativas son la base de todos los aspectos de la alfabetización estadística. Estas habilidades incluyen la comprensión de números, razones, porcentajes, tasas y fracciones.

- Faceta 11 (F11): Comprensión de textos y comunicación. Los textos que contienen estadísticas cívicas suelen ser muy densos ya que la información se presenta a partir de diferentes representaciones (números, tablas, gráficos, etc.). En ese sentido, los ciudadanos deben ser capaces de cambiar la representación de los datos para generar comprensión, es decir, realizar procesos de transnumeración (Batanero, Díaz, Contreras y Roa, 2013; Pfannkuch 
y Wild, 2004). Todo ello está relacionado con habilidad de comunicar conclusiones de manera comprensible y transparente.

Las facetas descriptas anteriormente conforman un marco conceptual para la Estadística Cívica. Y dicho marco es el que tuvimos en cuenta para el diseño de la tarea presentada y su posterior análisis.

\section{Metodología}

La tarea diseñada forma parte de la primera fase de una investigación de diseño (Molina, Castro y Castro, 2007) que busca, en una segunda etapa, analizar el aprendizaje de los estudiantes considerando la naturaleza sistémica del proceso de enseñanza y aprendizaje de Estadística. En esta primera fase, realizamos un análisis de contenido de la tarea para poder evaluar las potencialidades y limitaciones de la misma.

Dado que para realizar un análisis de contenido es necesario tener un marco de referencia que permita delinear unas categorías iniciales que guíen el análisis, hemos tomado las facetas de la Estadística Cívica, enunciadas por Engel (2019) y descritas previamente como categorías iniciales.

\subsection{Características del curso donde se implementa la tarea}

La investigación se desarrolla dentro del espacio curricular de Estadística en las carreras de Contador Público y Licenciatura en Ciencias de la Administración de la Facultad de Ciencias de la Administración de la Universidad Nacional de Entre Ríos. Dicho espacio es de cursado cuatrimestral con seis horas semanales y forma parte del ciclo profesional de dichas carreras ( $1^{\circ}$ cuatrimestre del tercer año). En el ciclo básico, los alumnos han cursado tres materias del área de Matemática, con un enfoque de enseñanza tradicional y en general, no cuentan con aprendizajes previos de estadística.

\subsection{Características generales de la tarea} La tarea forma parte de la primera unidad temática referida a Estadística Descriptiva y está pensada para trabajarla en grupos, con el objetivo de favorecer el debate e intercambio de opiniones. Cabe destacar que los alumnos ya vienen trabajando con actividades referidas al análisis, interpretación y evaluación crítica de gráficos estadísticos presentados en diferentes medios de comunicación.

Para el desarrollo de la tarea es central la manipulación e interpretación de los gráficos de burbujas dinámicos provistos por la fundación Gapminder (www.gapminder.org). Dicha fundación fue creada en 2005, por el médico y experto en salud pública Hans Rosling y provee datos de libre acceso recolectados por universidades, organismos de Naciones Unidas, agencias públicas, entre otros. Como se afirma en el sitio web de la fundación, su misión es luchar contra la ignorancia devastadora con una cosmovisión basada en hechos que todos puedan entender.

\subsection{Diseño de la tarea}

Teniendo en cuenta que los destinatarios son estudiantes universitarios que cuentan con conocimientos económicos y sociales, consideramos la noción de contexto significativo e importante, propuesta por Gal (2019), con el propósito de maximizar el sentido de la tarea, y promover el interés y participación de los alumnos. Desde la construcción de la tarea buscamos que los estudiantes:

- Aborden un fenómeno social y complejo, como es la relación entre el ingreso per cápita y la tasa de fertilidad (total) por país.

- Trabajen con datos recolectados por instituciones $y$ organismos oficiales.

- Analicen los datos desde diferentes perspectivas (agregándolos y desagregándolos).

- Describan y comparen distribuciones.

- Reconozcan la naturaleza omnipresente de la variación en el trabajo con datos.

- Analicen correlaciones y tendencias.

- Usen la evidencia empírica para debatir y discutir 
sobre las implicancias sociales, económicas, demográficas y políticas del fenómeno analizado.

- Comuniquen adecuadamente posiciones y conclusiones elaboradas a partir del análisis estadístico.

De los objetivos anteriores, se desprenden las ideas estadísticas fundamentales vinculadas a la tarea: datos, distribución, variación y correlación. En el Cuadro 1 se presenta el contenido de la misma.

Tomen entre 20 y 30 minutos para conocer la herramienta Gapminder y, en particular, para entender los gráficos de burbujas dinámicos. Pueden acceder desde: https://www.gapminder.org/tools/

Una vez que consideren que han comprendido ese tipo de gráficos:

1) Expresen claramente cómo se definen las variables ingreso per cápita y tasa de fertilidad e investiguen la confiabilidad y validez de los datos que utiliza la herramienta.

2) Exploren los gráficos de burbujas que resultan de considerar las variables ingreso per cápita y tasa de fertilidad, visualizando su comportamiento a través del tiempo. ¿Qué pueden observar? Fundamenten sus respuestas.

3) ¿Podría ser de interés para los gobiernos el análisis realizado en el punto anterior? ¿Por qué?

\section{Cuadro 1.}

Tarea diseñada.

\subsection{Algunas consideraciones didáctico-pedagógicas}

Antes de comenzar a resolver la actividad, los estudiantes deben comprender la herramienta de análisis estadístico Gapminder, lo cual requiere haber «probado» las diferentes posibilidades de visualización de datos que ofrece dicha herramienta $y$, en particular, las vinculadas a los gráficos de burbujas. Esto puede no resultar sencillo para algunos estudiantes, pero el trabajo en grupos facilita la ayuda entre ellos. Hay que tener claro que del conocimiento de la herramienta dependerá la riqueza de los análisis posteriores que hagan los estudiantes. Por lo que, de ser necesario, se podría destinar más tiempo a que interactúen con la herramienta.

Como los alumnos cuentan con conocimientos socioeconómicos, es de esperar que la definición de las variables no les presente inconvenientes, pero es importante que las definiciones queden explicitadas de manera precisa y clara para evitar malas interpretaciones. Deberán dar cuenta del ingreso per cápita como el cociente entre el Producto Bruto Interno (PBI) y el número total de habitantes; ${ }^{(1)}$ y la tasa de fertilidad como el número promedio de hijos que nacerían por mujer si todas vivieran hasta el final de sus años fértiles y dieran a luz de acuerdo con la tasa de fertilidad promedio para cada edad.(2) Asimismo, tendrán que investigar sobre la confiabilidad y validez de los datos provistos por Gapminder, lo cual implica conocer la reputación de las organizaciones e instituciones encargadas de la recolección de datos.

Desde el ítem 2, se expone a los estudiantes a la toma de decisiones. En principio, deberán decidir cuál variable representarán en el eje $x$ y cuál en el eje $y$, lo que los obliga (implícitamente) a construir una idea del tipo de relación que puede darse entre las variables. Tratándose de estudiantes que cuentan con aprendizajes matemáticos previos, puede resultarles sencillo decidir qué variable considerar en cada uno de los ejes. Sin embargo, deberán reconocer que no se trata de variables relacionadas funcionalmente, sino estadísticamente. Esto puede resultar una dificultad inicial que deberá ser tenida en cuenta por el docente.

Con el propósito de dar cuenta de posibles análisis que pueden realizar los estudiantes, presentamos algunas visualizaciones de gráficos de burbujas que, si bien son dinámicas, se muestran estáticas. Para observar las visualizaciones en forma dinámica, debajo de cada gráfico incluimos el enlace para acceder a cada uno (salvo los último dos que están completos). Cabe aclarar que los gráficos de burbujas no son gráficos de dispersión, ya que, si bien permiten observar relaciones entre variables, el tamaño de 
las burbujas es proporcional a una tercera variable que, en nuestro caso, refiere al tamaño poblacional (variable por defecto). Además, una cuarta variable se puede visualizar dinámicamente: con solo presionar el botón de play, el desarrollo de las variables se representa durante el período en el que se cuenta con datos. En el caso de las variables ingreso per cápita y tasa de fertilidad, el período es 1799 a 2018. Por otro lado, el uso de colores permite agregar y desagregar datos, de acuerdo con los objetivos del análisis.

Como se puede observar en los gráficos de las Figuras 1 y 2, es posible reconocer una tendencia decreciente, en la tasa de fertilidad, en países europeos desde el año 1850. Sin embargo, esa tendencia no se llega a observar en países de otros continentes por la dispersión que presentan las burbujas.
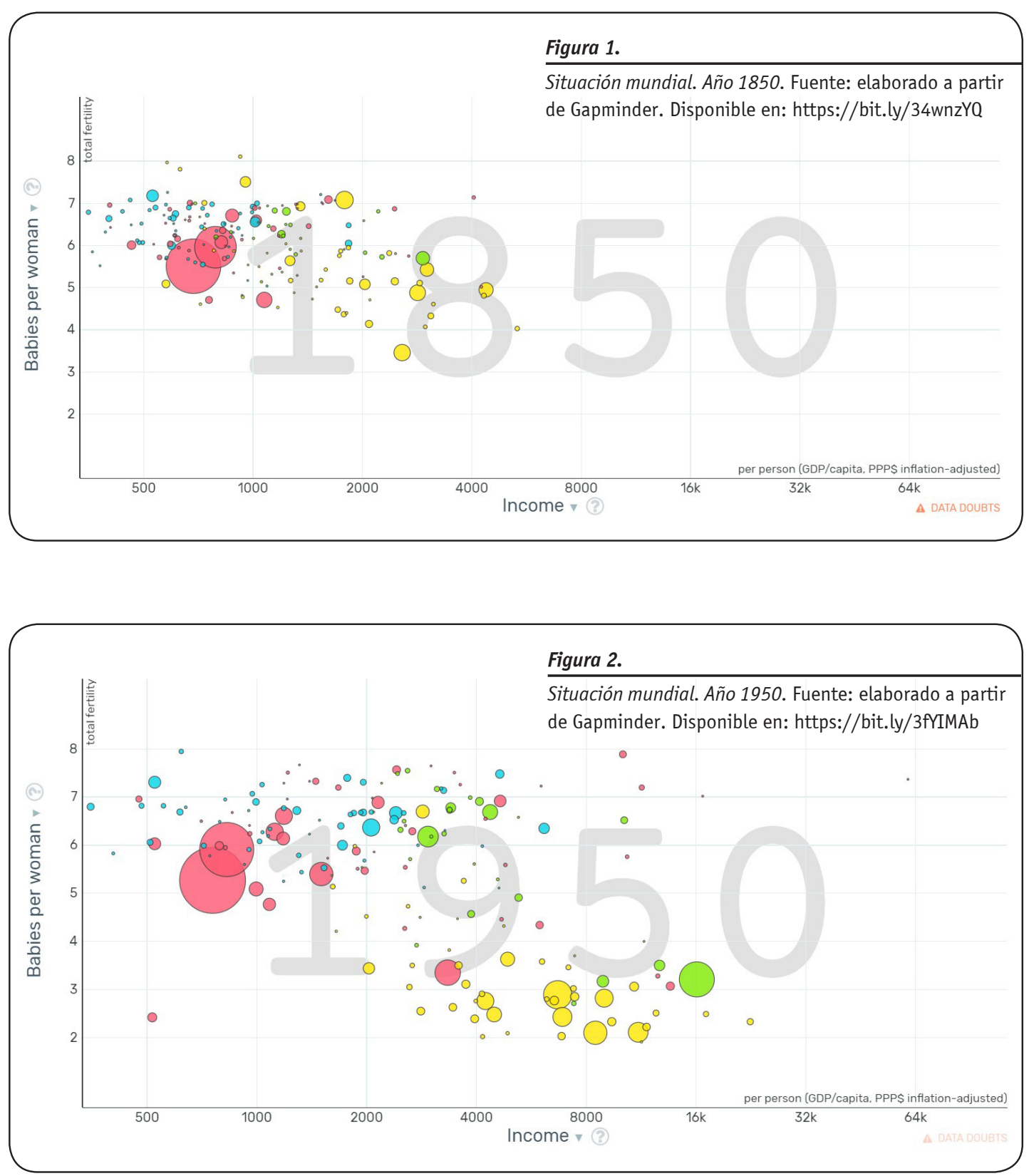
En el año 1990 (Figura 3) se comienza a vislumbrar la misma tendencia decreciente que en los países europeos, disminuyendo la variabilidad para 2018 (Figura 4). Es decir, los países con ingresos más elevados presentan tasas de fertilidad más bajas. Sin embargo, la tendencia no es tan marcada para los países africanos. ¿Por qué será?
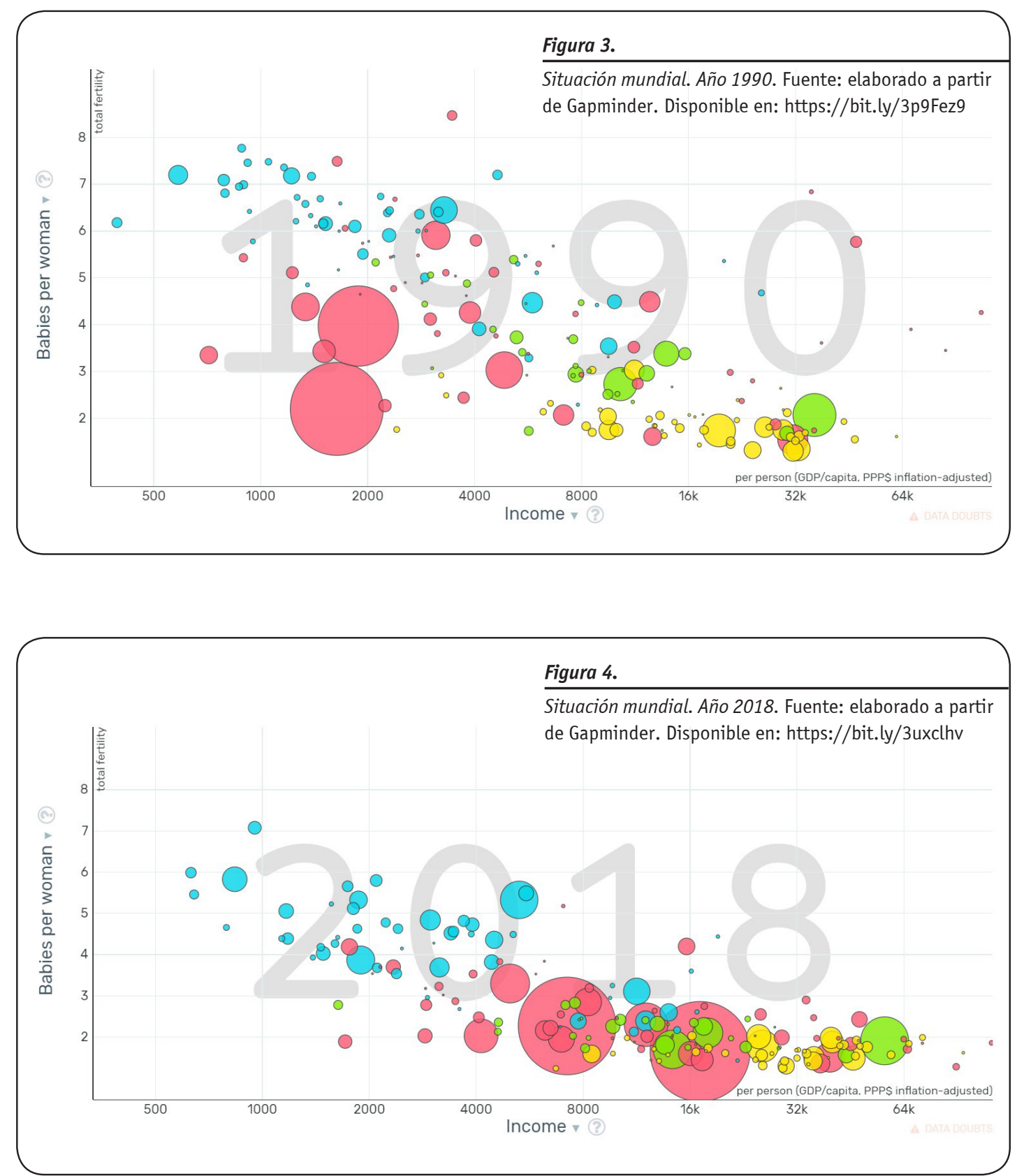
La dispersión que presentan los países africanos puede hacer que los alumnos desagreguen los datos para una exploración más detallada. Como muestra la Figura 5, en 1960, no se observa ninguna tendencia, sin embargo, ya para el año 1985 (Figura 6) algunos países comienzan a aumentar sus ingresos per cápita y disminuir sus tasas de fertilidad. Sin embargo, la Figura 7 muestra que en 2018 son pocos los países cuyas tasas de fertilidad se encuentran por debajo de tres (hijos promedio por mujer) habiendo efectivamente aumentado sus ingresos. Los estudiantes podrían realizar análisis complementarios - buscando información en la web-, para comprender el porqué de las situaciones que van analizando desde los gráficos.
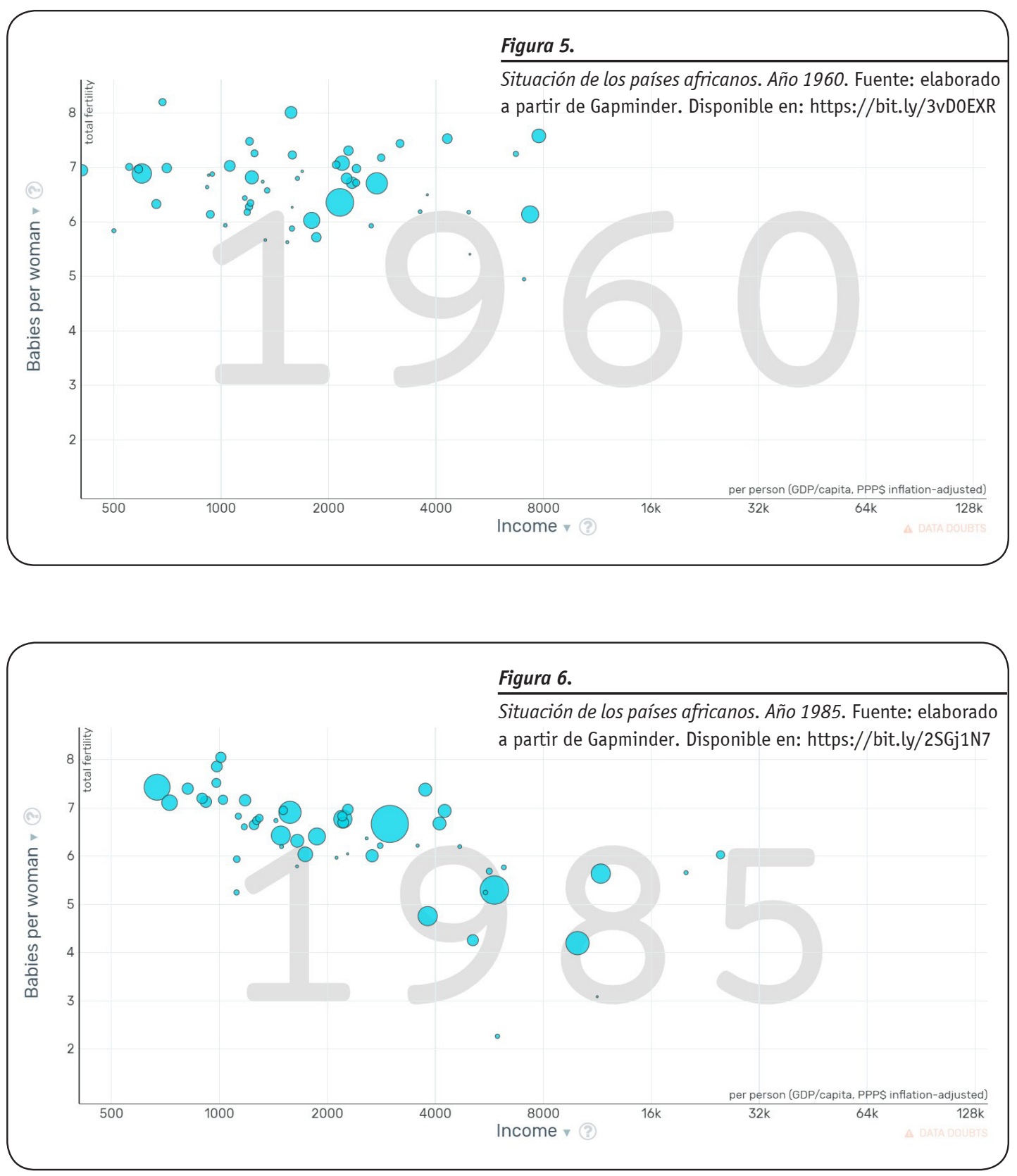


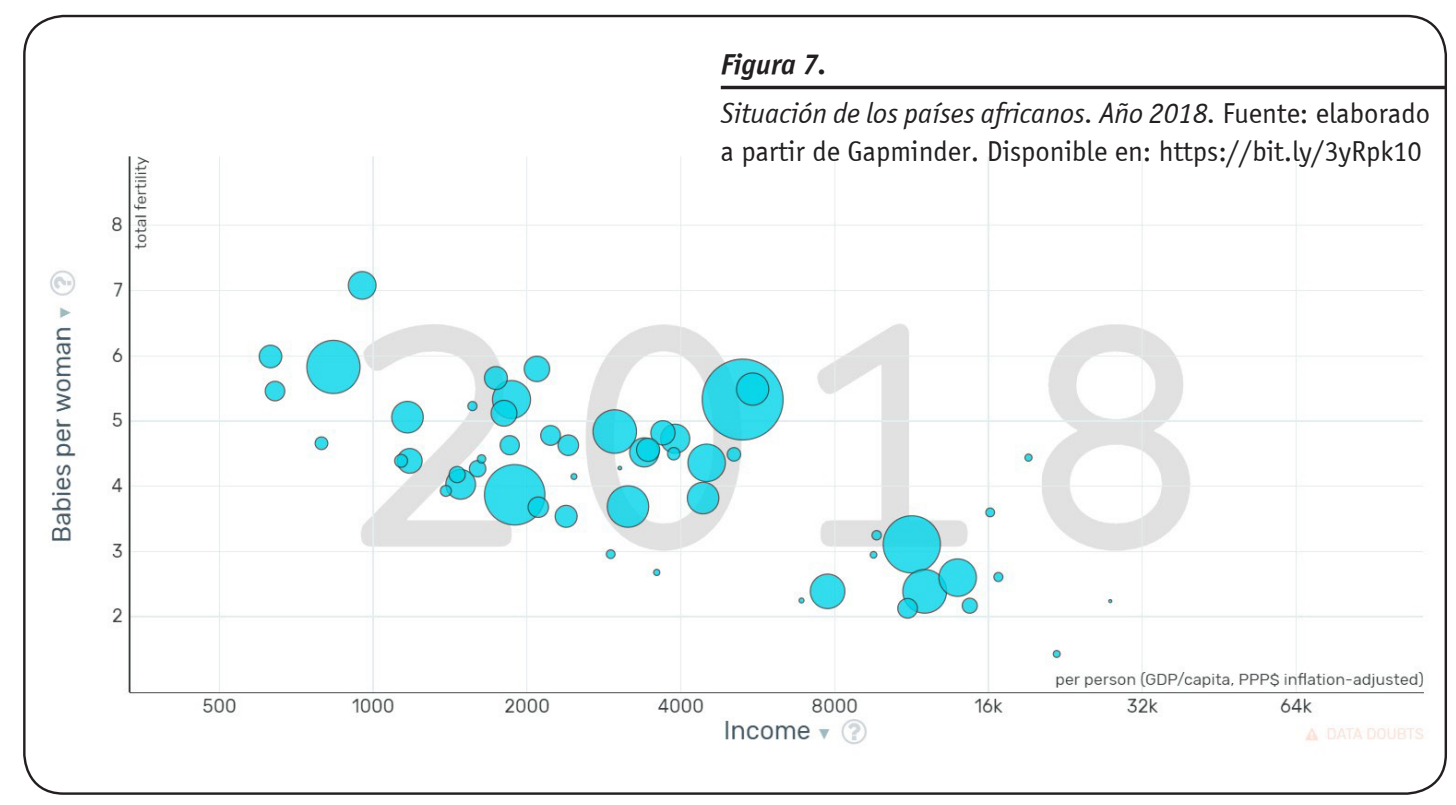

Para el fenómeno abordado en la tarea, China es un país que puede resultar particularmente interesante de analizar por la política de hijo único (vigente desde 1979) y que, en general, es conocida por los estudiantes. Los alumnos pueden observar cómo China logró descender la tasa de fertilidad, pasando de un promedio de siete hijos por mujer en la década de 1960 a menos de dos hijos en 2018 (Figura 8).

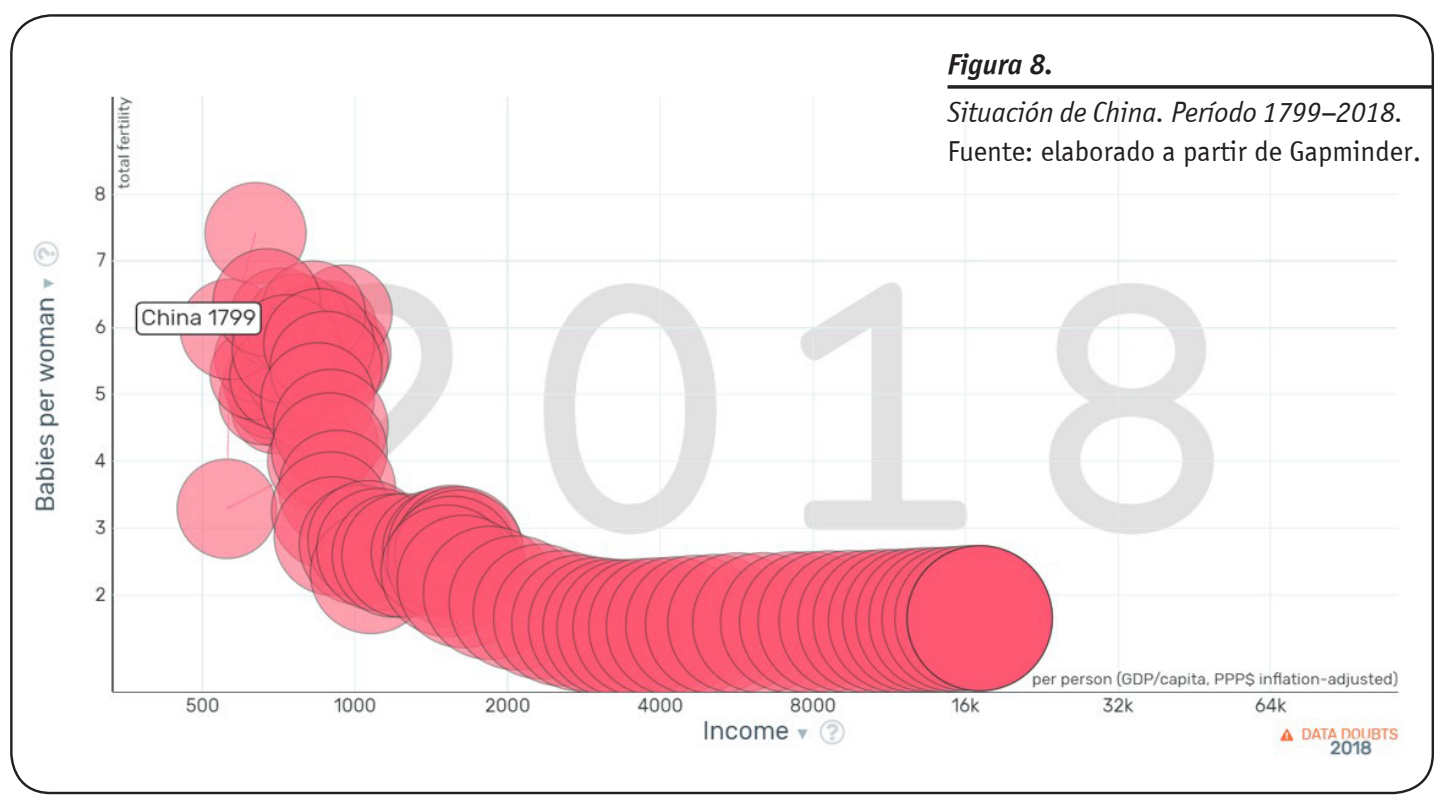


Los alumnos también podrían explorar la situación de su país, Argentina, y compararla con la tendencia mundial. Como se puede observar en la Figura 9, la tendencia es la misma, aunque es posible notar algunas variaciones, principalmente en la última década del siglo XIX. Si comparasen la situación de los últimos años de China y Argentina, se darían cuenta que los ingresos son similares pero la tasa de fertilidad es mayor en Argentina.

Esto también puede motivar a los estudiantes a buscar explicaciones de este tipo de situaciones -impensadas para muchos- promoviendo así actitudes de cuestionamiento y conciencia del fenómeno estudiado.

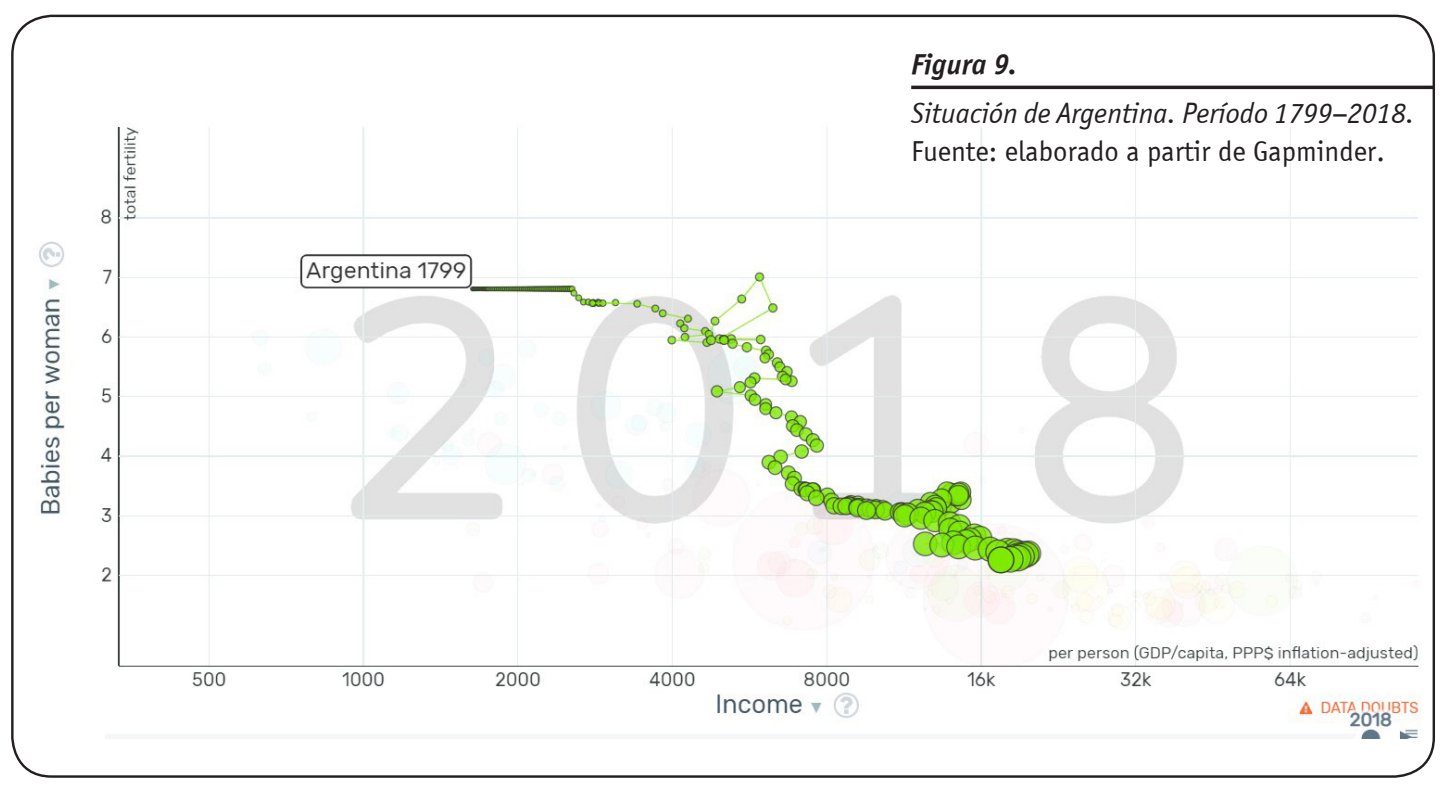

Los gráficos mostrados anteriormente constituyen posibles exploraciones que pueden realizar los estudiantes, pero de ninguna manera cubren todas las posibilidades de análisis.

A partir de lo realizado, los alumnos deberán responder la pregunta referida al interés que pueden tener los gobiernos acerca de los resultados y las conclusiones obtenidas (ítem 3). Aquí seguramente busquen información que complemente sus análisis. Probablemente surjan cuestiones vinculadas a las políticas de control de natalidad, las consecuencias económicas del envejecimiento de las poblaciones, la paradoja demográfico-económica, entre otras.

\section{Análisis de contenido de la tarea}

La tarea diseñada aborda un fenómeno multivariado vinculado, principalmente, con la economía y la demografía, y que surge de analizar la correlación entre el ingreso per cápita y la tasa de fertilidad por país. Según Engel (2019), analizar fenómenos socialmente relevantes «requiere una perspectiva interdisciplinaria, contiene elementos de conocimiento especiales $y$, por lo tanto, incluye elementos disposicionales no cognitivos» (p. 6).

En ese sentido, la definición que los estudiantes deberían hacer de cada variable (ítem 1), requiere la comprensión del significado contextual de cada. La primera se trata de una variable económica (ingreso per cápita) que los estudiantes conocen y utilizan 
en otras asignaturas, pero la segunda se trata de una variable demográfica (tasa de fertilidad), cuyo conocimiento puede ser impreciso o parcial. Así, la claridad del significado de cada una busca evitar lecturas incorrectas o malas interpretaciones. Por ejemplo, desde el inicio, los estudiantes tienen que tener claro que la tasa de fertilidad hace referencia a un promedio, no a una proporción. Como afirma Engel (2019), para modelar se debe tener una comprensión básica de los fenómenos que se modelan. Por otro lado, el análisis de la confiabilidad y validez de los datos habilita la evaluación crítica y promueve actitudes de cuestionamiento, y conciencia sobre el hecho de que el análisis de datos está íntimamente relacionado a las cuestiones metodológicas asociadas a la obtención de los mismos (Tauber, en prensa).

Para realizar las exploraciones de los gráficos de burbujas (ítem 2), los alumnos deben comenzar a tomar decisiones sobre qué analizar y cómo hacerlo, lo que habilita una trama de elementos cognitivos y disposicionales de la alfabetización estadística (Bolatti y Tauber, 2021). Esas decisiones los llevarán a analizar los modelos observados desde el conjunto de datos como un todo (Pfannkuch y Wild, 2004). Sin embargo, como afirma Engel (2019), las conclusiones por nivel de agregación pueden variar cuando los datos se agregan o desagregan dentro de subgrupos, como se puede observar en las orientaciones sobre el desarrollo de la tarea.

El análisis de los gráficos seguramente lleve a los alumnos a buscar información para lograr una mayor comprensión del fenómeno estudiado. De acuerdo con Gal (2004), la falta de familiaridad con el contexto en el que se han obtenido los datos dificulta imaginarse por qué ocurren ciertas diferencias o similitudes entre los modelos, o por qué pueden existir interpretaciones alternativas para los resultados sobre una asociación detectada entre ciertas variables, o por qué un análisis podría ser incorrecto. Es de esperar que los análisis que realicen los estudiantes habiliten una red de conceptos y de elementos cognitivos (Gal, 2019) que, en nuestro caso, están íntimamente asociados a las ideas estadísticas fundamentales vinculadas a la tarea: datos, distribución, variación y correlación. Los alumnos también deberán poner en juego habilidades para comunicar las conclusiones de manera adecuada y contextualizada, a través de procesos de transnumeración (Batanero, Díaz, Contreras y Roa, 2013; Pfannkuch y Wild, 2004). Cabe destacar que el desarrollo de la tarea está completamente mediado por la herramienta Gapminder. Por lo que son claves las habilidades de los alumnos en el uso de la tecnología para lograr que realicen análisis con riqueza estadística.

La pregunta referida al interés que pueden tener los gobiernos acerca de los análisis que realizaron (ítem 3) está relacionada con lo que afirma Engel (2019) respecto a que «los estudiantes y los ciudadanos deben poder comprender qué se puede y qué se debe hacer para debatiry abordar los problemas de las políticas de dominio» (p. 7). De acuerdo con Gal (2004), los alumnos tendrán que pensar en las consecuencias sociales, políticas y económicas que puede acarrear - para los gobiernos- esa correlación inversa entre los ingresos y la tasa de fertilidad. Esto los obliga a ponerse en el rol de tomadores de decisiones, utilizando elementos asociados al compromiso social y a la evaluación crítica de la información y de los conceptos estadísticos que la sustentan (Engel, 2019). En esta instancia hay que considerar lo planteado por Pfannkuch y Wild (2004), respecto a que no es usual encontrar estudiantes que esperen que la recolección y el análisis de los datos puedan confirmar su conocimiento personal de la situación. De hecho, los estudiantes generalmente ignoran los gráficos que ellos han construido y escriben sus conclusiones basadas en sus propias creencias. Uno de los elementos fundamentales del pensamiento estadístico es el reconocimiento de que los datos son necesarios para juzgar una situación (Batanero, Díaz, Contreras y Roa, 2013; Engel, 2019; Tauber, 2021).

En la Tabla 2 presentamos un resumen del análisis del contenido realizado -sobre cada parte de la tarea- a partir de las facetas de la Estadística Cívica descriptas por Engel (2019). 


\begin{tabular}{|c|c|c|c|}
\hline \multirow[t]{2}{*}{ Faceta (Fi) } & \multicolumn{3}{|l|}{ Tarea } \\
\hline & Ítem 1 & İtem 2 & Ítem 3 \\
\hline F1. Preparación para el compromiso social & & $x$ & $x$ \\
\hline F2. Evaluación crítica y reflexión & $\mathrm{x}$ & $x$ & $\mathrm{x}$ \\
\hline F3. Disposiciones & & $\mathrm{x}$ & $\mathrm{x}$ \\
\hline F4. Estadística y riesgo & & $x$ & \\
\hline F5. Modelos y representaciones & & $x$ & \\
\hline F6. Metodología y proceso de investigación & $x$ & $x$ & \\
\hline F7. Extensiones en el área de estadísticas oficiales & $x$ & & \\
\hline F8. Conocimiento social contextual & $x$ & $x$ & $x$ \\
\hline F9. TIC e investigación de la información & & $x$ & \\
\hline F10. Núcleo cuantitativo & & $x$ & \\
\hline F11. Comprensión de textos y comunicación & & $x$ & $\mathrm{x}$ \\
\hline
\end{tabular}

Tabla 2.

Facetas de la Estadística Cívica vinculadas a la tarea propuesta. Fuente: adaptada de Tauber (2021).

\section{Reflexiones finales}

Es imprescindible que los estudiantes reconozcan la importancia de la estadística para el estudio y comprensión de los fenómenos sociales. Y, en ese sentido, la enseñanza de la Estadística Cívica es clave para que los alumnos formen su propia posición, basada en la evidencia, que les permita participar -de manera competente e informada- en debates públicos sobre temas de relevancia social.

La tarea analizada conforma un ejemplo de cómo se puede trabajar con fenómenos sociales complejos en un contexto significativo para los estudiantes. El análisis de datos, las visualizaciones dinámicas y sus interpretaciones no solo están relacionadas con el conocimiento estadístico, sino que forman parte del conocimiento adquirido en muchas áreas y por lo tanto, requieren de una perspectiva interdisciplinaria.
El análisis de contenido muestra que es posible implementar una enseñanza de la Estadística orientada a formar ciudadanos y futuros profesionales estadísticamente cultos. Como dice Pinto Sosa (2020), la Educación Estadística tiene dos grandes desafíos:

1) Generar un cambio de paradigma que permita que la Estadística en la escuela se vincule más con lo que ocurre en la sociedad y 2) que los trayectos formativos se configuren de tal manera que todo profesionista logre comprender los problemas sociales que enfrentamos e incidir en su solución como ciudadano y desde su profesión. (p. 67) 


\section{Notas}

(1) https://www.gapminder.org/data/documentation/gd001/

(2) https://www.gapminder.org/data/documentation/gd008/

\section{Referencias bibliográficas}

Batanero, C., Díaz, C., Contreras, J.M. y Roa, R. (2013). El sentido estadístico y su desarrollo. Números, 83, 7-18. http://funes.uniandes.edu.co/3651/1/Batanero2013ElNumeros83.pdf Bolatti, F. y Tauber, L. (2021). Indicadores de la Estadística Cívica presentes en tareas que propician la cultura estadística. En J. Sagula (Ed.), Actas del II Simposio de Educación Matemática Virtual. Universidad Nacional de Luján. (en prensa).

Engel, J. (2019). Cultura estadística y sociedad. En J.M. Contreras, M.M. Gea, M.M. López-Martín y E. Molina-Portillo (Eds.), Actas del Tercer Congreso Internacional Virtual de Educación Estadística. https://digibug.ugr.es/bitstream/handle/10481/55028/engel_esp.pdf?sequence=1\&isAllowed=y Gal, I. (2004). Statistical Literacy: meanings, components, responsibilities. En: D. Ben-Zvi y J. Garfield (eds.), The challenge of developing statistical literacy, reasoning and thinking, pp. 47-78.

Gal, I. (2019). Understanding statistical literacy: About knowledge of contexts and models. En J. M. Contreras, M.M. Gea, M.M. López-Martín y E. Molina-Portillo (Eds.), Actas del Tercer Congreso Internacional Virtual de Educación Estadística.

Molina, M., Castro, E. y Castro, E. (2007). Teaching Experiments within Design Research. The International Journal of Interdisciplinary Social Sciences, 2(4), 435-440. D0I: https://doi. org/10.18848/1833-1882/CGP/v02i04/52362

Nicholson, J., Gal, I., y Ridgway, J. (2018). Understanding Civic Statistics: A Conceptual Framework and its Educational Applications. A product of the ProCivicStat Project. http://IASEweb.org/ISLP/PCS Pfannkuch, M. y Wild, C. (2004). Towards an understanding of statistical thinking. En: D. Ben-Zvi y J. Garfield (eds.), The challenge of developing statistical literacy, reasoning and thinking, pp. 17-45. Pinto Sosa, J.E. (2020). El cambio en el currículo en Educación Estadística en el ámbito universitario: dificultades y retos. Matemáticas, Educación y Sociedad, 3(2), 56-74.

Tauber, L. (2020). Dimensiones de la Alfabetización y el Pensamiento estadístico evidenciadas en una experiencia de evaluación sobre indicadores sociales. Actas del $4^{\circ}$ Encuentro Colombiano de Educación Estocástica (en prensa).

Tauber, L. (2021). Facetas de la Estadística Cívica Implícitas en una Experiencia de Enseñanza centrada en el Estudio de Indicadores Sociales. Paradigma, V. 42, Extra N 1, 89-117. D0I: 10.37618/ PARADIGMA.1011-2251.2021.p89-117.id1019 